\title{
Time-resolved four-wave mixing in InAs/InGaAs quantum-dot amplifiers under electrical injection
}

Borri, Paola; Langbein, Wolfgang Werner; Hvam, Jørn Märcher; Heinrichsdorff, F.; Mao, M.-H.; Bimberg, Dieter

Published in:

Applied Physics Letters

Link to article, DOI:

$10.1063 / 1.126038$

Publication date:

2000

Document Version

Publisher's PDF, also known as Version of record

Link back to DTU Orbit

Citation (APA):

Borri, P., Langbein, W. W., Hvam, J. M., Heinrichsdorff, F., Mao, M-H., \& Bimberg, D. (2000). Time-resolved four-wave mixing in InAs/InGaAs quantum-dot amplifiers under electrical injection. Applied Physics Letters, 76(11), 1380-1382. https://doi.org/10.1063/1.126038

\section{General rights}

Copyright and moral rights for the publications made accessible in the public portal are retained by the authors and/or other copyright owners and it is a condition of accessing publications that users recognise and abide by the legal requirements associated with these rights.

- Users may download and print one copy of any publication from the public portal for the purpose of private study or research.

- You may not further distribute the material or use it for any profit-making activity or commercial gain

- You may freely distribute the URL identifying the publication in the public portal 


\title{
Time-resolved four-wave mixing in InAs/InGaAs quantum-dot amplifiers under electrical injection
}

\author{
P. Borri, ${ }^{a}$, b) W. Langbein, ${ }^{\text {a) }}$ and J. M. Hvam \\ Research Center COM, The Technical University of Denmark, Building 349, DK-2800 Lyngby, Denmark \\ F. Heinrichsdorff, M.-H. Mao, and D. Bimberg \\ Institut für Festkörperphysik, Technische Universität Berlin, Hardenbergstr. 36, 10623 Berlin, Germany
}

(Received 21 October 1999; accepted for publication 19 January 2000)

\begin{abstract}
Time-resolved four-wave mixing in an InAs/InGaAs/GaAs electrically pumped quantum-dot amplifier is measured at room temperature for different applied bias currents going from optical absorption to gain of the device. The four-wave mixing signal from $140 \mathrm{fs}$ pulses shows a transition from a delayed photon-echo response in the absorption regime to a prompt free polarization decay in the gain regime. This corresponds to a pronounced reduction of the dephasing time from $250 \mathrm{fs}$ at zero bias to less than $50 \mathrm{fs}$ at the maximum applied current. The four-wave mixing response at transparency of the device shows a composite structure with both photon echo and free-polarization decay. This is a signature of the digital occupation number in quantum dots, resulting at transparency in a signal from dots occupied with either zero or two excitons corresponding to absorption or gain of the dot ground state. (C) 2000 American Institute of Physics.
\end{abstract}

[S0003-6951(00)02211-7]

The homogeneous optical linewidth of semiconductor quantum dots is a key subject currently under discussion. ${ }^{1}$ Besides the fundamental interest in the underlying scattering mechanisms, the expected superior performances of quantum-dot lasers rely on a delta-like density of states, which is affected by the homogeneous and inhomogeneous broadenings. Presently the inhomogeneous broadening due to size and/or strain fluctuations in real $\operatorname{In}(\mathrm{Ga})$ As quantumdot (QD) structures is believed to exceed the homogeneous broadening, but recent improvements in growth technology have reduced the observed linewidth to $21 \mathrm{meV}$ in InAs/ InGaAs QDs, ${ }^{2}$ and the question arises: what is the intrinsic homogeneous linewidth limit in real QD lasers? In a recent letter $^{3}$ we reported the dephasing time $T_{2}$ in InAs/InGaAs QDs at room temperature, which determines the homogeneous linewidth (full width at half maximum) by $\gamma$ $=2 \hbar / T_{2}$. The dephasing time was determined by femtosecond time-resolved four-wave mixing (TR-FWM) in heterodyne detection, ${ }^{4}$ with the dots embedded in a $400-\mu \mathrm{m}$-long and $8-\mu \mathrm{m}$-wide ridge-waveguide structure. A homogeneous linewidth of $\sim 5 \mathrm{meV}$, weakly dependent on the optical excitation intensity was found and attributed to phonon interaction. The sample was a P-I-N junction for electrical injection and was processed with tilted facets to avoid lasing, but no electrical bias was applied.

In this letter we report the FWM response of the same sample in the presence of electrically injected carriers, for different values of bias currents ranging from absorption to gain in the ground-state transition of the dots. The purpose is to investigate the homogeneous linewidth in a real QD laser at working condition. Details of the sample structure and of the experimental setup can be found in Ref. 3 .

\footnotetext{
${ }^{a}$ Present address: Lehrstuhl für Experimentelle Physik EIIb, Universität Dortmund, Otto-Hahn Str. 4, 44227 Dortmund, Germany.

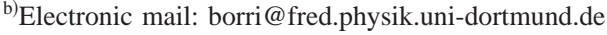

The bias current was tuned from 0 to $20 \mathrm{~mA}$, the latter corresponding to full saturation of the optical gain in the dot ground state transition at $1.08 \mu \mathrm{m}$. The maximum modal gain is $\sim 9 \mathrm{~cm}^{-1}$, which is consistent with lasing operation observed on a 1.5-mm-long cavity. ${ }^{5}$ Amplified spontaneous emission spectra at the output of the sample are shown in Fig. 1 at different bias current as indicated. A strongly inhomogeneously broadened $(\sim 60 \mathrm{meV})$ ground-state transition is observed at low bias current, in agreement with photoluminescence measurements ${ }^{6}$ which also reveal a separation of $\sim 110 \mathrm{meV}$ between the dot ground-state transition and the wetting layer. With increasing current, filling of high-energy states and wetting layer transitions at $\sim 1.0 \mu \mathrm{m}$ is observed. Note that the low energy tail of the ground-state transition slightly broadens with increasing current, indicating an increase of the homogeneous linewidth.

The FWM response of a strongly inhomogeneously broadened system is a photon echo in real time, and shows in

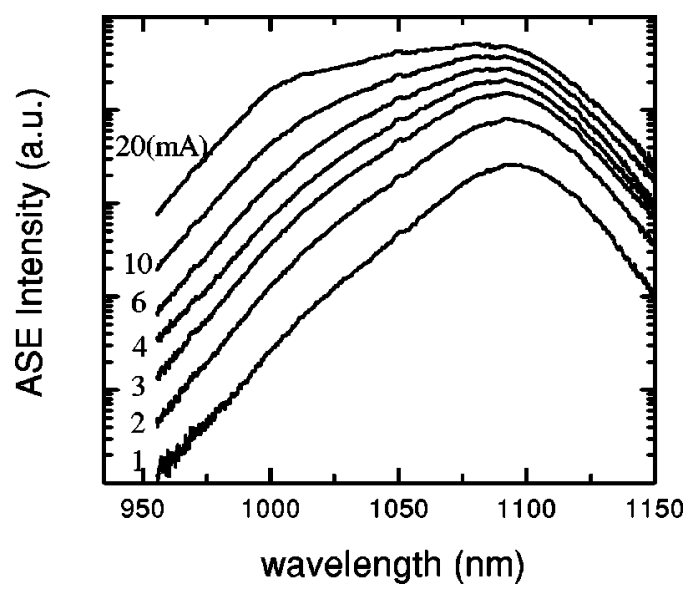

FIG. 1. Amplified spontaneous emission spectra at different bias currents, as indicated. 


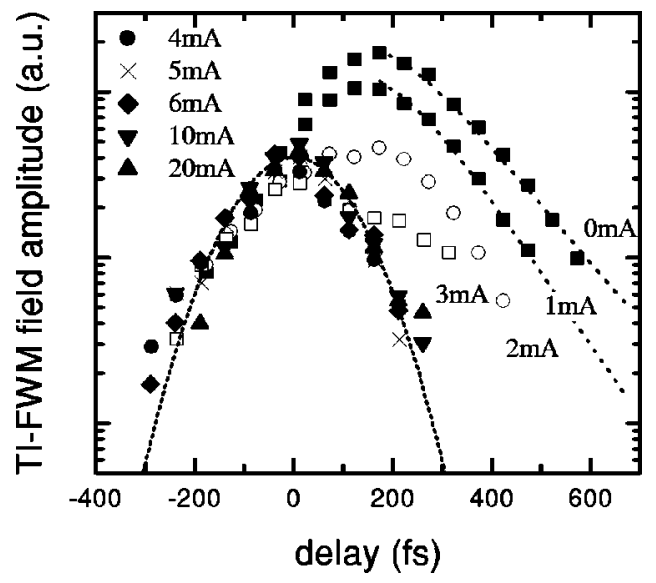

FIG. 2. Time-integrated FWM field amplitude at different bias currents vs delay time between the two exciting pulses. The dotted lines at 0 and $1 \mathrm{~mA}$ are numerical simulations of the FWM decay, corresponding to a dephasing time of 250 and $200 \mathrm{fs}$, respectively. The dashed line is the calculated instantaneous response.

the time-integrated signal a delayed maximum as a function of the delay time between the two exciting pulses. ${ }^{7}$ In a homogeneously broadened system the signal is a freepolarization decay that occurs in real time promptly after the second pulse and has the time-integrated maximum at zero delay time. ${ }^{7}$ The FWM response of the sample at zero bias current $^{3}$ is a photon echo with a delayed maximum of the time-integrated intensity, in agreement with a dominant inhomogeneous broadening within the spectral pulsewidth. In Fig. 2 the time-integrated FWM (TI-FWM) field amplitude is shown for different bias currents. Up to $2 \mathrm{~mA}$ bias current the TI-FWM has a delayed maximum as in a photon echo. The dotted lines in Fig. 2 at 0 and $1 \mathrm{~mA}$ are numerical simulations to the FWM decay at positive delay, obtained by solving the optical Bloch equations of an inhomogeneously broadened system for a Gaussian pulse of 140 fs intensity width. ${ }^{3}$ Dephasing times of $250 \mathrm{fs}$ at $0 \mathrm{~mA}$ and of $200 \mathrm{fs}$ at $1 \mathrm{~mA}$ are used in the simulations and agree with a simple exponential fit of the curves at long positive delays. The device reaches transparency (zero net number of stimulated transitions) close to $3 \mathrm{~mA}$ bias current, as measured by pump-probe experiments. ${ }^{8}$ At this bias the TI-FWM response has, at zero delay, the minimum value compared to all other bias currents, as expected at optical transparency for a nonlinear signal induced by a density grating. However, the delay dependence is quite peculiar, with the appearance of a delayed signal after the initial decay. When further increasing the bias current from 4 up to $20 \mathrm{~mA}$ a fast decay of the TI-FWM signal, with no delayed maximum, is observed which follows an instantaneous response (dashed line in Fig. 2). Comparisons with the numerical simulations give an upper limit of $50 \mathrm{fs}$ for the dephasing time at currents larger than $4 \mathrm{~mA}$.

The transition from a photon echo to a free-polarization decay with increasing bias current is more pronounced in TR-FWM as shown in Fig. 3. Here, the real-time dynamics of the FWM field is resolved by tuning the delay of the reference pulse in the heterodyne detection ${ }^{3,4}$ while keeping a fixed delay time of 120 fs between the two exciting pulses. Zero reference delay corresponds to full overlap of reference and second exciting pulse. The TR-FWM signal is clearly Downloaded 05 Feb 2010 to 192.38.67.112. Redistribution subject

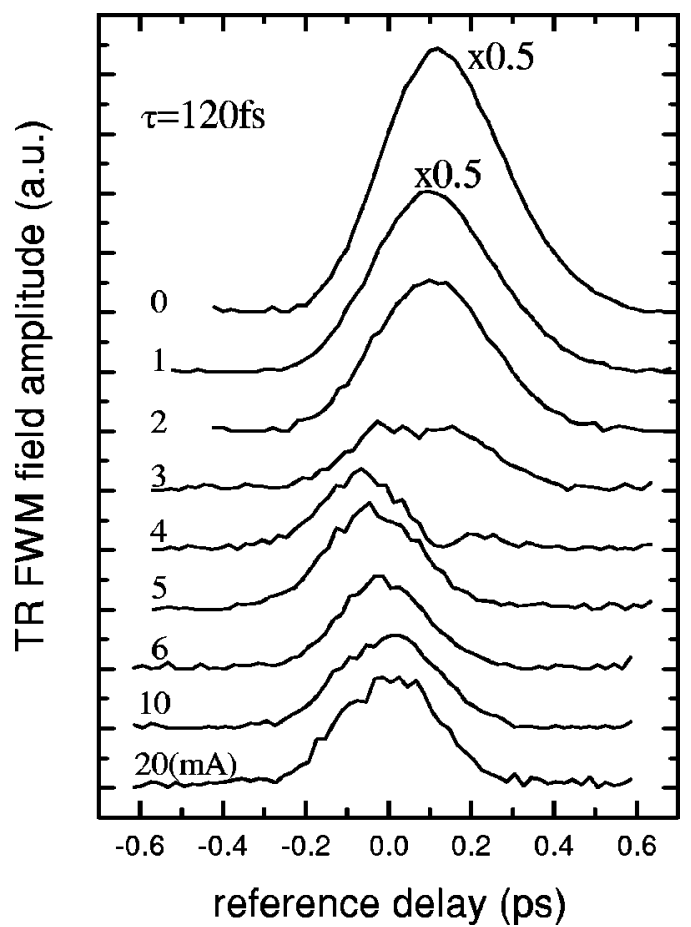

FIG. 3. Time-resolved FWM at fixed delay time of $120 \mathrm{fs}$ between the two exciting beams for different bias currents, as indicated. A double structure around transparency is clearly resolved.

delayed in real time at 0,1 , and $2 \mathrm{~mA}$ bias current, as expected for a photon echo, while it shows an instantaneous response above $5 \mathrm{~mA}$. However, around transparency the occurrence of a double structure with both a delayed and an instantaneous component is visible.

These observations can be explained as follows. The shortening of the dephasing in the presence of electrically injected carriers indicates an increasing carrier-carrier scattering of the QD ground state. Below $2 \mathrm{~mA}$ the average occupation number is less than one exciton per dot, and carrier-carrier scattering inside the same dot is unlikely. We thus suggest that scattering with the thermalized carriers occupying the wetting layer is responsible for the slight reduction of the dephasing time. Note that a significant number of carriers are present at thermal equilibrium in the wetting layer due to its high density of states, even if $k T$ is smaller than the confinement energy in the QDs. At transparency, the average occupation number is one exciton per dot and the optically induced net density change is zero so that there should be no FWM signal. However, due to the discrete value of the occupation number in QDs, an average occupation of one exciton per dot is made out of a distribution of dots occupied by zero, one, and two excitons. Even dots with more than two excitons per dot should be present in a small fraction. The empty dots will then contribute to a photonecho signal, as in the absorption case, with a shortened dephasing time due to scattering with carriers in the wetting layer. In the dots occupied by two or more excitons, carriercarrier scattering inside the dot is possible, and leads to a strongly decreased dephasing time. For bias currents above transparency, most dots are occupied by two or more excitons, and the FWM signal is dominated by the fast response. Note that $T_{2}<50$ fs corresponds to $\gamma>26 \mathrm{meV}$, comparable to the pulse spectral width in the experiment and therefore to AIP license or copyright; see http://apl.aip.org/apl/copyright.jsp 
corresponding to a dominant homogeneously broadened system within the pulse spectrum. We should mention that differential transmission experiments performed in the gain region, which probe the population inversion lifetime, showed $\sim 100$ fs recovery time of the spectral hole burned by the pump pulse. ${ }^{8}$ This indicates the occurrence of fast carriercarrier scattering also in the energy relaxation dynamics, possibly due to intradot Auger scattering. However the population lifetime is still significantly above the measured dephasing, which is thus dominated by elastic scattering processes.

In conclusion, we have shown that the increase of bias current on a QD amplifier leads to a significant shortening of the dephasing time, corresponding to a homogeneous linewidth already comparable to presently achieved inhomogeneous distributions of QD ensembles. This implies a dominant homogeneous broadening behavior in QD lasers, as also recently reported. ${ }^{9}$ Moreover, the FWM response around transparency shows clear features of the discrete values of the occupation number in QDs, different from what is observed in bulk and QWs. ${ }^{10}$

The authors thank J. Mørk for many useful discussions and J. Bjørn at GIGA for mounting the sample. This work was supported by the Danish Technical Research Council in the framework of SCOOP and by DFG in the framework of SFB 296. The authors acknowledge Tele Danmark R\&D for the donation of part of the equipment.

${ }^{1}$ D. Bimberg, M. Grundmann, and N. N. Ledentsov, in Quantum Dot Heterostructures (Wiley, England, 1999).

${ }^{2}$ K. Nishi, H. Saito, S. Sugou, and J.-S. Lee, Appl. Phys. Lett. 74, 1111 (1999).

${ }^{3}$ P. Borri, W. Langbein, J. Mørk, J. M. Hvam, F. Heinrichsdorff, M.-H. Mao, and D. Bimberg, Phys. Rev. B 60, 7784 (1999).

${ }^{4}$ P. Borri, W. Langbein, J. Mørk, and J. M. Hvam, Opt. Commun. 169, 317 (1999).

${ }^{5}$ F. Heinrichsdorff, Ph.D. thesis, Institut für Festkörperphysik, Technische Universität Berlin, Hardenbergstr. 36, 10623 Berlin, 1997 (unpublished).

${ }^{6}$ F. Heinrichsdorff, M.-H. Mao, N. Kirstaedter, A. Krost, D. Bimberg, A. O. Kosogov, and P. Wagner, Appl. Phys. Lett. 71, 22 (1997).

${ }^{7}$ J. Shah, in Ultrafast Spectroscopy of Semiconductors and Semiconductor Nanostructures (Springer, Berlin, 1996), Chap. 2.

${ }^{8}$ P. Borri, W. Langbein, J. M. Hvam, M.-H. Mao, F. Heinrichsdorff, and D. Bimberg, Conference on Lasers and Electro-Optics OSA Technical Digest (Optical Society of America, Washington DC, 1999), pp. 321-322; P. Borri, W. Langbein, J. Mørk, J. M. Hvam, M.-H. Mao, F. Heinrichsdorff, and D. Bimberg, European Conference on Optical Communication Technical Digest, Paris, 1999 (unpublished), Vol. II, p. 74.

${ }^{9}$ M. Sugawara, K. Mukai, and Y. Nakata, Appl. Phys. Lett. 74, 1561 (1999).

${ }^{10}$ M. Hofmann, S. D. Brorson, J. Mørk, and A. Mecozzi, Appl. Phys. Lett. 68, 3236 (1996). 